\title{
MicroRNA-340 Induces Apoptosis and Inhibits Metastasis of Ovarian Cancer Cells by Inactivation of NF- $\mathrm{KB} 1$
}

\author{
Peiquan Li Yuxin Sun Qing Liu \\ Department of Gynaecological Oncology, Ren Ji Hospital, School of Medicine, Shanghai JiaoTong \\ University, Shanghai, People's Republic of China
}

\author{
Key Words \\ MicroRNA-340 • Ovarian cancer • Proliferation • Invasion • EMT • NF-KB1
}

\begin{abstract}
Aims: Aberrant expression of microRNA-340 (miR-340) has been frequently reported in some cancers excluding ovarian cancer (OC). The role and its molecular mechanism of miR-340 in OC have not been reported. Methods: Real-time PCR was performed to detect the expression of miR-340 in OC cell lines. MiR-340 mimic and negative control were transfected into OC cells and the effects of miR-340 on the cell proliferation, cell cycle, apoptosis and metastasis were investigated by Brdu-ELISA assay, flow cytometry, qRT-PCR, Transwell and ELISA assays. Furthermore, protein level of NF-kB1 was measured by Western blotting. Meanwhile, luciferase assays were performed to validate NF-KB1 as miR-340 target in OC cells. Results: In this study, we explored the effects of miR-340 overexpression on apoptosis, invasion and EMT in OC cells. The mRNA level of miR-340 in OC cell lines and tissues was evidently reduced. The miR-340 mimic was transiently transfected into OC cells using Lipofectamine ${ }^{\mathrm{TM}} 2000$ reagent. Subsequently, the Brdu-ELISA results showed that introduction of miR-340 inhibited cell proliferation. Our data also demonstrated that miR-340 mimic arrested cell cycle progression and promoted apoptosis of OC cells. In addition, miR-340 overexpression could also inhibit invasion and EMT of OC cells. qRT-PCR were used to determined the expressions of matrix metalloproteinase-2 and -9 (MMP-2 and -9) in OC cells. Next, we found that NF-KB1 expression was evidently reduced by up-regulation of miR-340. Bioinformatics analysis predicted that the NF- $\mathrm{kB1}$ was a potential target gene of miR-340. Luciferase reporter assay further confirmed that miR-340 could directly target the $3^{\prime}$ UTR of NF-KB1. Moreover, overexpression of NF-KB1 in OC cells transfected with miR-340 mimic partially reversed the inhibitory of miR-340 mimic. Conclusion: miR-340 induced cell apoptosis and inhibited metastasis in OC cells by downregulation of NF-KB1.
\end{abstract}

P. Li and Y. Sun are the co-first authors.

Dr. Qing Liu,

KARGER
Department of Gynaecological Oncology, Ren Ji Hospital School of Medicine Shanghai JiaoTong University, No.145 Shandong Rod, Huangpu District, Shanghai 200127, (People's Republic of China)

Tel.+86-021-53882038, Fax +86-021-53882038,E-Mail liuqinghuangpu@163.com 


\section{Introduction}

Ovarian cancer (OC) is still a major cause of morbidity and mortality, with little change in survival rates in the recent 30 years. Recently, previous reports have demonstrated that several genes are closely related to human ovarian cancer [1]. However, the precise molecular mechanisms of ovarian cancer are not defined yet. As far as we know, the mechanisms of OC are revealed by focusing on known genes, but focusing on unknown microRNAs (miRNAs) may also lend insight into the biology of OC.

Many studies have reported that miRNAs are small (about 21-23 nucleotide in length) [2]. Moreover, miRNAs, a new layer of gene-regulation mechanism, can regulate cellular proliferation, differentiation and apoptosis, as well as cancer initiation and progression [3, 4]. Actually, many studies have showed that miRNAs were differentially regulated in diverse cancer types such as colorectal cancer [5], breast cancer [6], lung cancer [7]. Recently, a lot of reports showed that many miRNAs are aberrantly expressed in multiple cancers such as OC [8-11]. The miR-17-5p was overexpressed in OC cells, and up-regulation of miR-17-5p significantly promoted proliferation, migration, invasion and EMT of OC cells by targeting PTEN signaling [12]. Niu et al. found that miR-205 expression was associated with motility of OC by targeting ZEB1 [13]. MiR-661 promoted proliferation of OC cells through directly targeting INPP5] [14]. These three miRNAs act as oncogene, whereas some tumor suppressor miRNAs were also studied in OC. For example, Overexpression of miR-30d reversed TGF$\beta 1$-induced EMT of OC cells by decreasing Snail expression [15]. The level of miR-127 was markedly down-regulated in OC tissues, and overexpression of miR-127 inhibited proliferation of OC cells by targeting cyclin G1 (CCNG1) [16]. MiR-302a is involved in the suppression of SDC1 to inhibit the tumorigenicity of OC cells [17].

MiR-340 has been involved in regulation of multiple biological processes such as cell proliferation, apoptosis, migration and invasion [18]. In recent years, miR-340 was considered as a tumor suppressor and down-regulated in prostate, esophageal and non-small cell lung cancers [19-21]. Wei et al. found that miR-340 overexpression inhibited proliferation and invasive properties of prostate cancer cells by targeting high-mobility proup nucleosomebinding domain 5 (HMGN5) [19]. Furthermore, miR-340 was also reduced in esophageal cancer cells (ESCCs), and functioned as a tumor suppressor to inhibit growth, colony formation and invasion of ESCCs by down-regulation of phosphoserine aminotransferase 1 (PSAT1) [20]. Fernandez and his colleague found that miR-340 also functioned as a tumor suppressor through inhibiting proliferation and inducing apoptosis in non-small cell lung cancer cells by regulation of p27 [21].

In this paper, down-regulation of miR-340 was frequently observed in OC cell lines. Upregulation of miR-340 inhibited cell proliferation, arrested cell cycle and induced apoptosis of OC cells. In addition, miR-340 overexpression could also inhibit invasion and EMT of OC cells. Moreover, we found that NF- $\kappa \mathrm{B} 1$ was the direct target of miR-340 in OC and confirmed that miR-340 functioned as a tumor suppressor by down-regulation of NF- $\kappa B 1$. Therefore, our results showed critical roles for miR-340 in the pathogenesis of OC and suggested its potential application in tumor treatment.

\section{Materials and Methods}

\section{Cell culture and human tissues}

Human OC cell lines such as OVCAR3, CAOV3, HO-8910, ES-2, A2780 and an immortalized normal human fallopian tube epithelial cell line FTE187 cells were purchased from American Type Culture Collection (ATCC, Manassas, VA, USA). OVCAR3, CAOV3, HO-8910, ES-2 and A2780 were cultured in Dulbecco's modified Eagle's medium (DMEM) (Gibco Co., USA) including 10\% fetal bovine serum (FBS) (Gibco Co., USA), $100 \mathrm{U} / \mathrm{ml}$ penicillin and $100 \mathrm{ug} / \mathrm{ml}$ streptomycin. FTE187 cell line was maintained in cell culture medium consisting of 1:1 Medium 199 and MCDB105 medium (Sigma-Aldrich) with 10\% FBS and $10 \mathrm{ng} / \mathrm{ml}$ EGF (Sigma-Aldrich). All cells were cultured at $37^{\circ} \mathrm{C}$ in a humidified atmosphere of $5 \% \mathrm{CO}_{2}$ on 


\section{Cellular Physiology Cell Physiol Biochem 2016;38:1915-1927

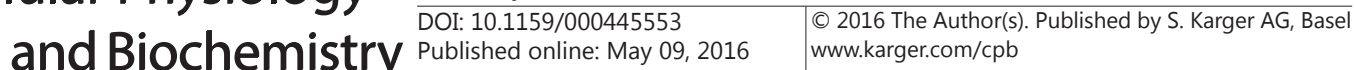 \\ Li/Sun/Liu: MiR-340 Inhibits Growth and Metastasis of OC Cells by NF-kB1 Inactivation}

$0.1 \%$ gelatin-coated culture flasks. Ten pairs of human ovarian carcinoma and their corresponding adjacent normal tissues were collected from Ren Ji Hospital Affiliated to Shanghai JiaoTong University School of Medicine. The specimens were immediately frozen in liquid nitrogen and then stored at $-80{ }^{\circ} \mathrm{C}$ for analysis. Prior informed consent was obtained, and the study protocol was approved by the Ethics Committee of Ren Ji Hospital Affiliated to Shanghai JiaoTong University School of Medicine.

MiRNA transient transfection

To enhance the expression of miR-340 in CAOV3 and A2780 cells, both cells were transfected with miR-340 mimic, which served as the miR-340 group. CAOV3 and A2780 cells transfected with miR-negative control (miR-NC) were used as miR-NC group. One day before transfection, cells at about 40 to $60 \%$ confluency were changed to the antibiotic-free media. After $24 \mathrm{~h}$, cells were transfected with $50 \mathrm{nM}$ miR-340 mimic using Lipofectamin ${ }^{\mathrm{TM}} 2000$ reagent (Invitrogen, USA) following manufacturer's protocol.

Reverse transcription polymerase chain reaction

Total RNA of CAOV3 and A2780 cells was extracted by using Trizol reagent (Life Technologies, CA). Two microgram RNA was used for gene-specific reverse transcription polymerase chain reaction (RT-PCR) using one-step RT-PCR kit (Qiagen, Venlo, the Netherlands) following the manufacturer's protocols. The following primers were used: miR-340, forward: 5'- CCGGGATCCGCAAACTCAGCTTTAC-3' and reverse: 5'- CGGAATTCGTGGCGACCGTGATACC-3'; E-cadherin, forward 5'-TACACTGCCCAGGAGCCAGA-3' and reverse: 5'-TGGCACCAGTGTCCGGATTA-3'; N-cadherin, forward: 5'-CGAATGGATGAAAGACCCATCC-3' and reverse: 5'-GGAGCCACTGCCTTCATAGTCAA-3'; Vimentin, forward: 5'-GCTGAATGACCGCTTCGCCAACT'-3' and revere: 5'-GCTCCCGCATCTCCTCCTCGTA-3'; MMP-2, forward: 5'- CTGCGGTTTTCTCGAATCCA-3' and reverse: 5'- GGGTATCCATCGCCATGCT-3'; MMP-9, forward: 5'- CCCTGGAGACCTGAGAACCA-3' and revere: 5'- CCACCCGAGTGTAACCATAGC-3'; U6, forward: 5'-CTCGCTTCGGCAGCACA-3' and reverse: 5'-AACGCTTCACGAATTTGCGT-3'; GAPDH, forward: 5'-GAGTCAACGGATTTGGTCGTATTG-3' and reverse: 5'-CCTGGAAGATGGTGATGGGATT-3'. U6 snRNA and GAPDH mRNA were used to normalize. Each sample was assessed in triplicate.

\section{Cell proliferation assay}

To study the role of miR-340 mimic in proliferation of CAOV3 and A2780 cells, $5 \times 10^{3}$ cells were seeded in 96-well plate and allowed to grow for $24 \mathrm{~h}$ in complete medium. The medium was then removed and the cells were transfected with miR-340 mimic or miR-NC for $24 \mathrm{~h}$ at $37^{\circ} \mathrm{C}$. Cell Proliferation ELISA-BrdU (colorimetric) Kit (Roche Diagnostics, USA) was used to detect the cells proliferation according to the manufacturer's protocols.

\section{Cell cycle analysis}

To detect cell cycle distribution, the CAOV3 and A2780 cells were transfected with miR-340 mimic for $24 \mathrm{~h}$. After transfection, CAOV3 and A2780 cells were collected by trypsinization, washed with ice-cold PBS, and fixed in ice-cold 70\% methanol overnight. Then, cells were centrifuged, resuspended in ice-cold phosphate buffer saline (PBS), and incubated with RNase (Sigma, USA) for $30 \mathrm{~min}$ at $37{ }^{\circ} \mathrm{C}$, and then were incubated with propidium iodide (PI; Sigma, USA) at room temperature for $30 \mathrm{~min}$. The analyses of cell cycle distribution were performed by FACScan flow cytometer (BD Biosciences, USA).

\section{Annexin V-FITC/PI analysis}

CAOV3 and A2780 cells were transfected with miR-340 mimic for $24 \mathrm{~h}$. After transfection, cells were harvested and washed twice in PBS and double-stained with Annexin V-FITC and PI by using the Annexin V-FITC Apoptosis Detection Kit (BD Biosciences, USA) following the manufacturer's protocols. Then, each sample was quantitatively analyzed at $488 \mathrm{~nm}$ emission and $570 \mathrm{~nm}$ excitation by FACSCalibur flow cytometer (BD Biosciences, USA).

Transwell invasion assay

Transwell matrigel invasion assay using Transwell chambers (8-mm pore size; Corning, USA) precoated with Matrigel (BD Biosciences, USA) that included extracellular matrix proteins was used to evaluate cell invasion. In brief, after serum-starved for $24 \mathrm{~h}, 2 \times 10^{5}$ cells were suspended in $100 \mu \mathrm{l}$ serum-free DMEM, 


\section{Cellular Physiology Cell Physiol Biochem 2016;38:1915-1927 \\ \begin{tabular}{c|c|c|} 
DOI: 10.1159/000445553 & $\begin{array}{l}\text { O 2016 The Author(s). Published by S. Karger AG, Basel } \\
\text { www.karger.com/cpb }\end{array}$
\end{tabular} \\ Li/Sun/Liu: MiR-340 Inhibits Growth and Metastasis of OC Cells by NF-kB1 Inactivation}

and then were seeded in the top chamber, and $600 \mu \mathrm{l}$ DMEM containing 10\% FBS was added to the lower chamber. After $24 \mathrm{~h}$ incubation at $37{ }^{\circ} \mathrm{C}$ in a $5 \% \mathrm{CO}_{2}$ atmosphere, cells that remained in the upper surface of the membrane were removed by cotton swabs and penetrating cells were fixed in methanol, and then stained with $0.1 \%$ crystal violet. Cell invasion was quantified by counting cells on the lower surface using phase contrast microscopy.

\section{Western blot analysis}

To extract the proteins, CAOV3 and A2780 cells were washed twice in cold PBS, and then lysed in RIPA lysis buffer (Beyotime Institute of Biotechnology, China) with protease inhibitor cocktail (Merk, Germany). The protein concentration of cell lysates was quantified by BCA Kit (Beyotime Institute of Biotechnology, China), and equal quantities (50 $\mu \mathrm{g}$ ) of proteins were separated by SDS-PAGE on $10 \%$ gels, and then transferred to a polyvinylidene fluoride (PVDF) membrane (Millipore, USA). The membranes were blocked in 5\% shimmed milk diluted with Tri Buffered Saline Tween-20 (TBST) (in mmol/L: Tris-HCl 20, NaCl 150, $\mathrm{PH} 7.5,0.1 \%$ Tween 20) at room temperature for $1 \mathrm{~h}$ and incubated overnight at $4{ }^{\circ} \mathrm{C}$ with primary antiNF-kB1 antibody (1:1000; Cell Signaling Technology Inc, USA). The membranes were then incubated with a goat anti-rabbit IgG conjugated to horseradish peroxidase secondary antibody $(1: 1000$; Cell Signaling Technology Inc, USA) for $2 \mathrm{~h}$. The proteins were visualized using ECL-plus reagents (Beyotime Institute of Biotechnology, China). The density of the bands was measured using the Image J software (USA), and values were normalized to the densitometric values of GAPDH (1:1000; Cell Signaling Technology Inc, USA) in each sample.

\section{Measurement of MMP-2 and MMP-9 levels}

Enzyme-linked immunosorbent assay (ELISA) kits (USCN, USCN life science, Wuhan, China) was used to determine the levels of MMP-2 and -9 in the culture supernatants based on the manufacturer's instructions.

Luciferase reporter assay

CAOV3 and A2780 cells $\left(2 \times 10^{5} /\right.$ well) were seeded in 24-well plates and incubated overnight before transfection. Cells were cotransfected with pMIR-NF-кB1-3'UTR wild-type or mutant reporter plasmid, miR-340 mimic or miR-NC, and pRL-SV40 renilla plasmid (Promega, USA) using Lipofectamine 2000. At 48 $\mathrm{h}$ after co-transfection, both firefly and renilla luciferase activities were quantified using a dual luciferase reporter system (Promega, USA) following the manufacturer's protocols. Each treatment was performed in triplicate in three independent experiments.

\section{Statistical analysis}

All statistical analyses were performed using GraphPad Prism 5.0 (GraphPad Software, Inc., USA). Data from each group were expressed as mean \pm standard error of the mean (S.E.M.) and statistically analyzed by Student's $t$ test. Differences were considered statistically significant at a $p$ value of $<0.05$.

\section{Results}

The level of miR-340 was decreased in OC cell lines

Many reports have showed that miR-340 was reduced in prostate, esophageal and nonsmall cell lung cancers [15-17]. However, the level of miR-340 in OC is still unclear. Therefore, the level of miR-340 was determined by RT-PCR in five OC cell lines such as OVCAR3, CAOV3, HO-8910, ES-2 and A2780 and an immortalized normal human fallopian tube epithelial cell line FTE187. Our findings demonstrated that expression of miR-340 was evidently reduced in all OC cell lines compared to that in normal human fallopian tube epithelial cell line FTE187 (Fig. 1A). Moreover, the levels of miR-340 in the OC tissues were also reduced and evidently lower in comparison to the adjacent tissues (Fig. 1B). Next, we found that NF- $\mathrm{BB} 1$ was predicted by using the online database (TargetScan 6.2) to be a direct target of miR-340. Then, among these OC cell lines, CAOV3 and A2780 cells were used to study further. Our results showed that the protein level of NF- $\mathrm{\kappa B} 1$ in CAOV3 and A2780 cells was significantly increased compared with FTE187 cell (Fig. 1C). 
Fig. 1. The altered expression of miR340 and NF- $\mathrm{KB} 1$ in OC cell lines. (A) The relative level of miR-340 in OC cell lines and FTE187 cell line by RT-PCR. (B) Relative miR-340 expression levels in $\mathrm{OC}$ tissues and their corresponding adjacent normal tissues. (C) NF- $\kappa$ B1 protein level in CAOV3 and A2780 cells compared with FTE187 cell were determined by Western blotting. GAPDH was detec-
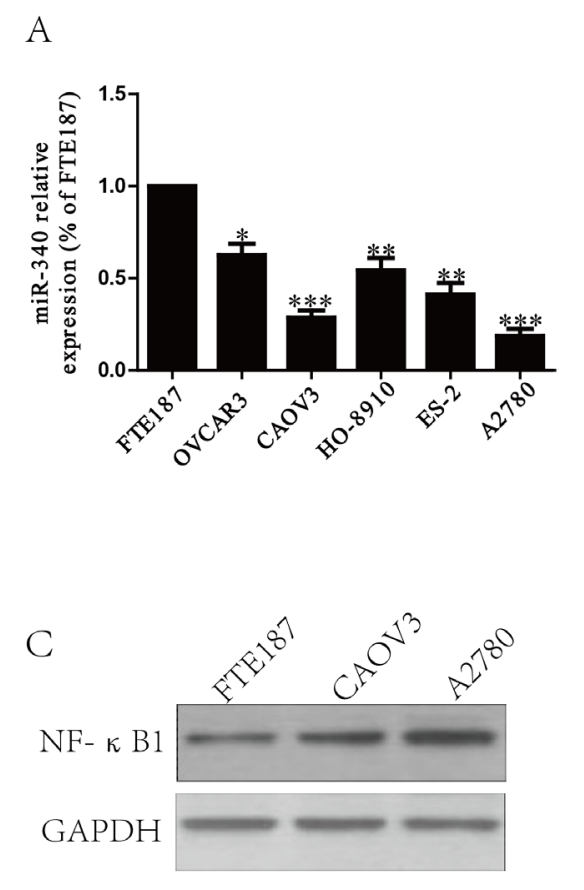
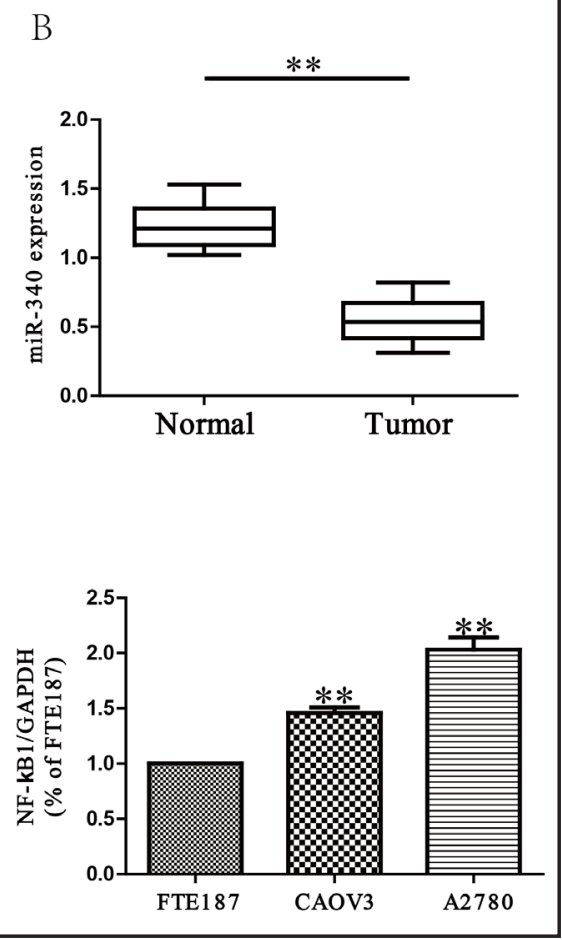

ted as a loading control. All data are presented as mean $\pm \mathrm{SEM}, \mathrm{n}=6 .{ }^{*} \mathrm{P}<0.05,{ }^{* *} \mathrm{P}<0.01,{ }^{* * *} \mathrm{P}<0.1$ vs. FTE187 or normal tissues.

Introduction of miR-340 inhibited proliferation, arrested cell cycle and induced cell apoptosis of CAOV3 and A2780 cells

However, the decreased expression of miR-340 in OC cell lines indicated that miR-340 might act as a tumor suppressor in OC. We found that level of miR-340 was evidently upregulated after transfection with miR-340 mimic compared to miR-NC group (Fig. 2A). Our data indicated that we could availably enhance miR-340 level in CAOV3 and A2780 cells. To investigate the role of miR-340 in proliferation of OC cells, CAOV3 and A2780 cells were transfected with miR-340 mimic or miR-NC. Results from Brdu-ELISA assay showed that overexpression of miR-340 clearly suppressed the viabilities of CAOV3 and A2780 cells (Fig. 2B). These findings indicated that up-regulation of miR-340 had available anti-proliferative effect in both CAOV3 and A2780 cells.

Because miR-340 mimic obviously inhibited proliferation of CAOV3 and A2780 cells, we speculated that introduction of miR-340 could arrest cell cycle of OC cells. Our data confirmed this tentative by flow cytometry. We demonstrated that up-regulation of miR-340 dramatically enhanced the percentage of cells in the G1/G0 peak and reduced the percentage of cells in the S peak in both CAOV3 and A2780 cells compared with cells transfected with miR-NC (Fig. 2C). Thus, overexpression of miR-340 might inhibit OC cell proliferation by impeding the G1/S cell cycle transition.

For studying whether pro-apoptotic effect was involved in miR-340-induced antiproliferative effect, we use flow cytometry analysis to detect the total apoptosis rates of CAOV3 and A2780 cells. We found that the number of apoptotic CAOV3 and A2780 cells was significantly higher in miR-340 group than that in miR-NC group (Fig. 2D).

MiR-340 overexpression restrained the invasive capacities of OC cells

To understand whether introduction of miR-340 had a negative role in invasion of OC cells, we transfected miR-340 mimic into CAOV3 and A2780 cells, and the invasive abilities were evaluated by Transwell invasion assay. We found that the number of CAOV3 


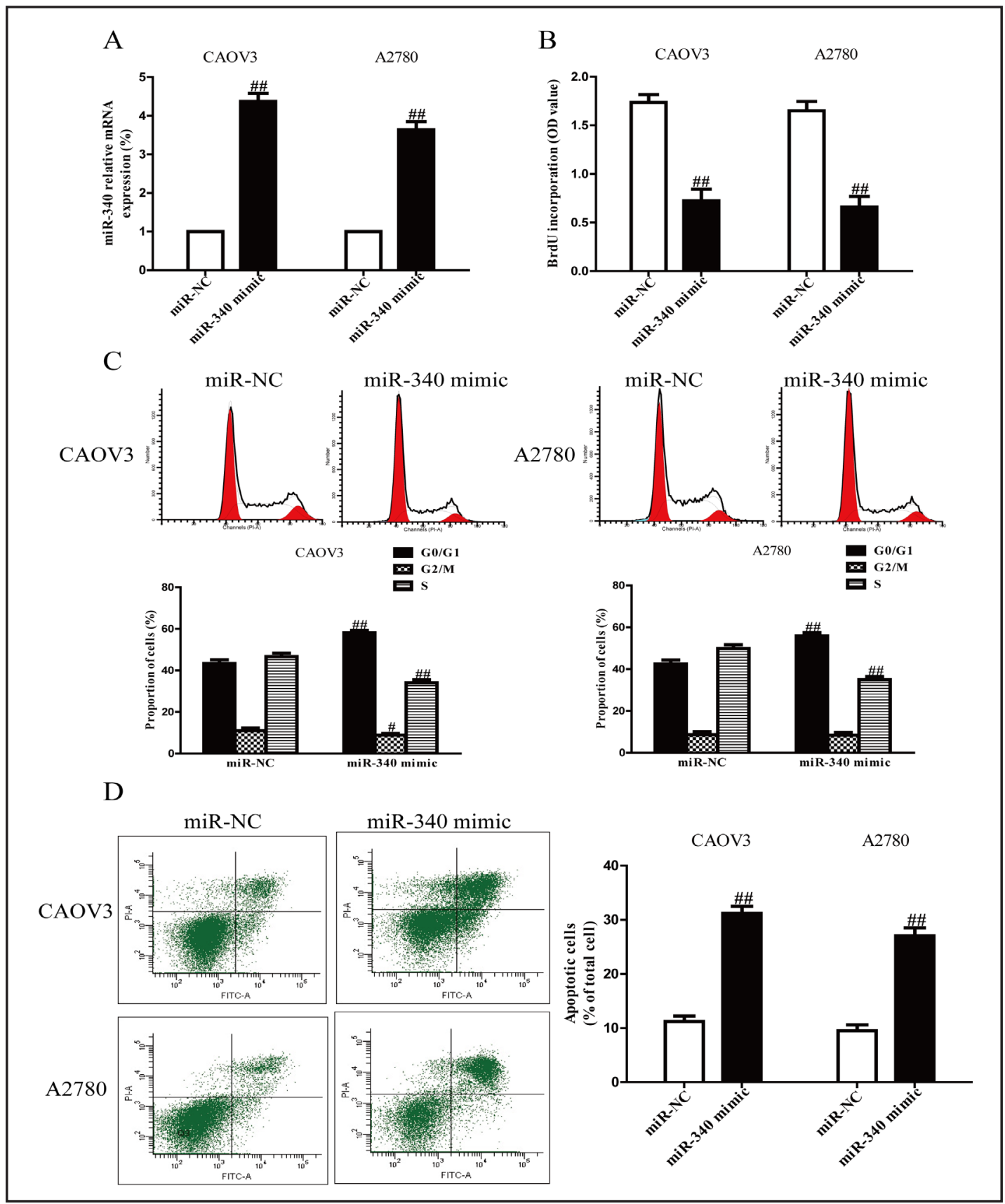

Fig. 2. Effects of miR-340 overexpression on proliferation, cell cycle and apoptosis of CAOV3 and A2780 cells. CAOV3 and A2780 cells were transfected with miR-340 mimic or miR-NC. (A) The mRNA levels of miR340 in CAOV3 and A2780 cells were detected by RT-PCR. (B) Cell proliferation was assessed by BrdU-ELISA assay. (C) Cell cycle was detected by flow cytometry. (D) Apoptosis of CAOV3 and A2780 cells was measured by flow cytometric analysis of cells labeled with Annexin-V/PI double staining. All data are presented as mean \pm SEM, $n=6$. ${ }^{\#} \mathrm{P}<0.05,{ }^{\# \#} \mathrm{P}<0.01$ vs. miR-NC.

and A2780 cells invading through the Transwell membrane was evidently less in miR-340 group compared to miR-NC group (Fig. 3). Our data indicated that up-regulation of miR-340 suppressed the invasion of OC cells. 
Fig. 3. Overexpression of miR340 suppressed invasion of OC cells. CAOV3 and A2780 cells were transfected with miR-340 mimic and miR-NC, and then seeded in the top chamber. After $24 \mathrm{~h}$, the CAOV3 and A2780 cells that invading through the membrane were stained and quantified. Data are expressed as percentage of miRNC. Data represent means \pm SEM,

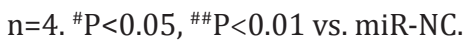

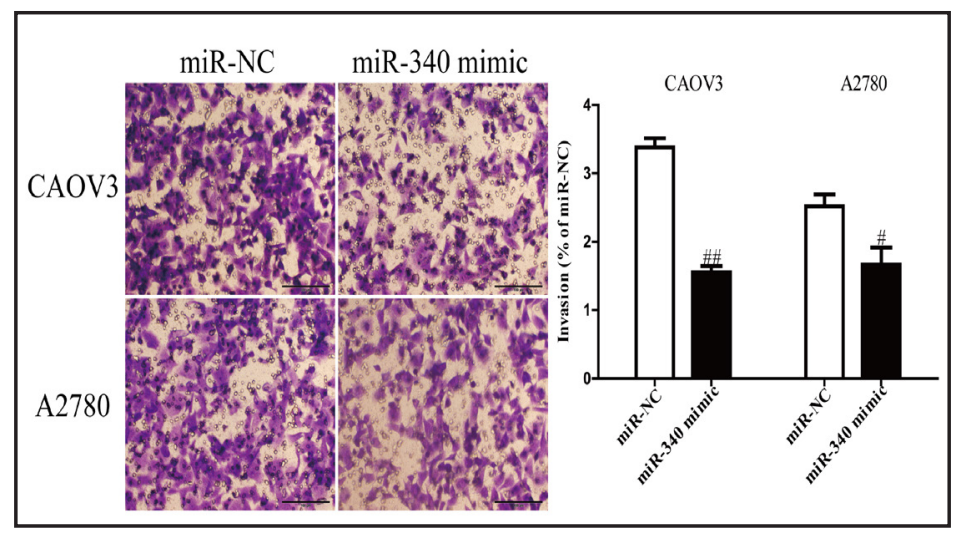

Fig. 4. Overexpression of miR-340 decreased expressions and secretions of MMP-2 and -9. CAOV3 and A2780 cells were transfected with miR-340 mimic and miR-NC. (A) Levels of MMP-2 and -9 were detected by ELISA assay in the culture supernatants of cultured CAOV3 and A2780 cells. (B) The mRNA levels of MMP-2 and -9 were examined by qRT-PCR. All data are presented as mean \pm SEM, $n=6$. ${ }^{\# \#} \mathrm{P}<0.01$, ${ }^{\# \# \# P}<0.001$ vs. miRNC.

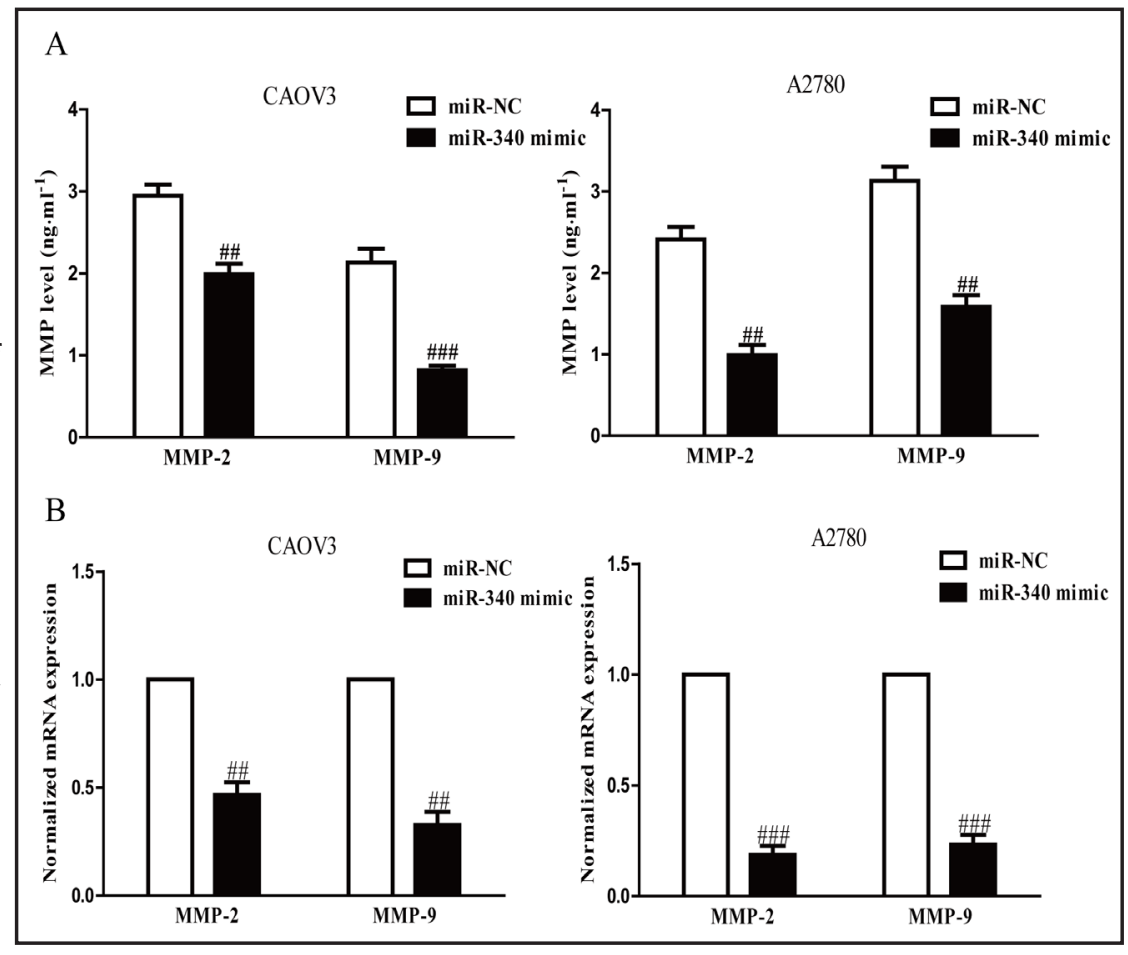

Effects of miR-340 overexpression on expressions of MMP-2 and -9 in OC cells

MMPs may be responsible for the impaired invasion of miR-340 mimic-transfected cells. To confirm this hypothesis, we determined the levels of MMP-2 and MMP-9 in the culture supernatants by ELISA. Our data showed that levels of MMP-2 and -9 in the culture supernatants were significantly decreased in CAOV3 and A2780 cells transfected with miR340 mimic (Fig. 4A). Besides, we further detected the levels of MMP-2 and -9 at the mRNA levels by qRT-PCR. Consistent with the results of ELISA assay, the overexpression of miR-340 operated a distinct reduction in MMP-2 and -9 expressions at the mRNA (Fig. 4B) levels. Our results suggested that up-regulation of miR-340 suppressed the migratory ability of CAOV3 and A2780 cells by down-regulation of MMP-2 and -9 .

Effects of miR-340 overexpression on EMT-related molecules of OC cells

Next, to know whether EMT contributed to inhibition of OC cell invasion by upregulation of miR-340, we studied the effects of miR-340 overexpression on the mRNA levels of EMT markers in CAOV3 and A2780 cells by qRT-PCR. Introduction of miR-340 in CAOV3 and A2780 cells led to up-regulation of E-cadherin that was an epithelial marker, and down- 


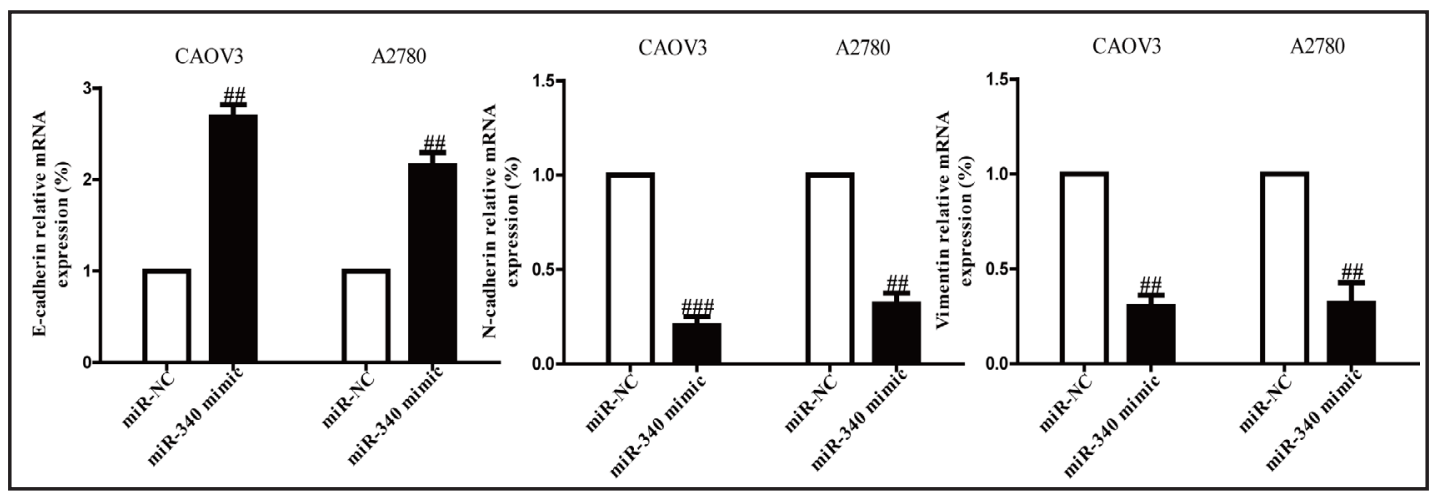

Fig. 5. Overexpression of miR-340 regulated the mRNA levels of EMT-related molecules in OC cells. CAOV3 and A2780 cells were transfected with miR-340 mimic and miR-NC. The mRNA levels of E-cadherin, N-cadherin and Vimentin were determined by qRT-PCR in CAOV3 and A2780 cells. GAPDH was detected as a loading control. All data are presented as mean $\pm \mathrm{SEM}, \mathrm{n}=6 .{ }^{\# \#} \mathrm{P}<0.01$, ${ }^{\# \#} \mathrm{P}<0.001$ vs. miR-NC.

Fig. 6. $N F-\kappa B 1$ was a direct target of miR-340. CAOV3 and A2780cells were transfected with miR340 mimic or miRNC. (A) The protein expression of NF- $\mathrm{KB} 1$ was determined by Western blot. GAPDH was detected as a loading control. (B) Schematic representation of NF- $\mathrm{KB} 1$ 3'UTRs showing putative miRNA target site. (C) The analysis of the relative luciferase activities of NF- $\kappa B 1$ WT, NF- $\kappa B 1-M U T$ in OC cells. All data are presented as mean \pm SEM, $n=6$. ${ }^{~} \mathrm{P}<0.05$, ${ }^{\# \#} \mathrm{P}<0.01$ vs. miR-NC.
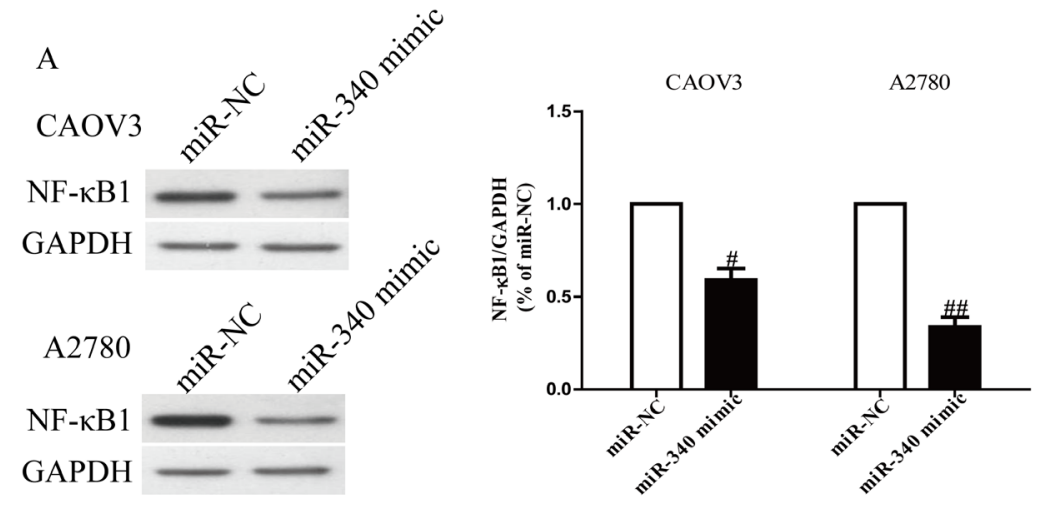

B

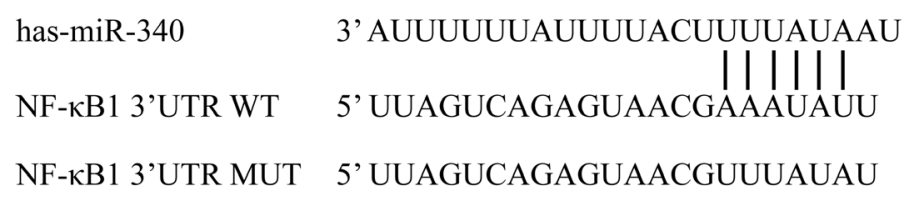

$\mathrm{C}$

CAOV3

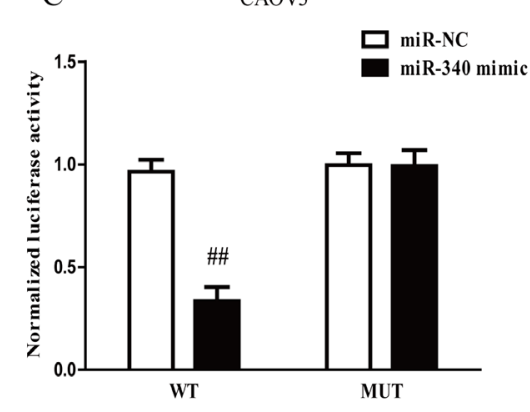

regulation of $\mathrm{N}$-cadherin and Vimentin that were the mesenchymal markers at mRNA levels (Fig. 5). Taken together, our results indicated that up-regulation of miR-340 could markedly restrain the invasive ability of OC cells partly by regulation of EMT. 
Fig. 7. Overexpression of NF- $\kappa \mathrm{B} 1$ partially rescued miR-340-inhibited cell proliferation and invasion in OC cells. CAOV3 and A2780 cells were transfected with either miR-340 mimic with or without pCDNA-NF- $\kappa$ B1 vector. (A) The protein expression of NF- $\mathrm{kB} 1$ was determined by Western blot. $\alpha$-tubulin was detected as a loading control. (B) Cell proliferation was assessed by BrdU-ELISA assay. (C) The invasion of CAOV3 and A2780 cells was assessed by Transwell assay. (D) The mRNA levels of E-cadherin, $\mathrm{N}$-cadherin and Vimentin were determined by qRT-PCR in CAOV3 and A2780 cells. GAPDH was detected as a loading control. All data are presented as mean \pm SEM, $\mathrm{n}=6$. ${ }^{\# \#} \mathrm{P}<0.01,{ }^{\# \# \#} \mathrm{P}<0.001$ vs. miR-340 mimic + pcDNA.
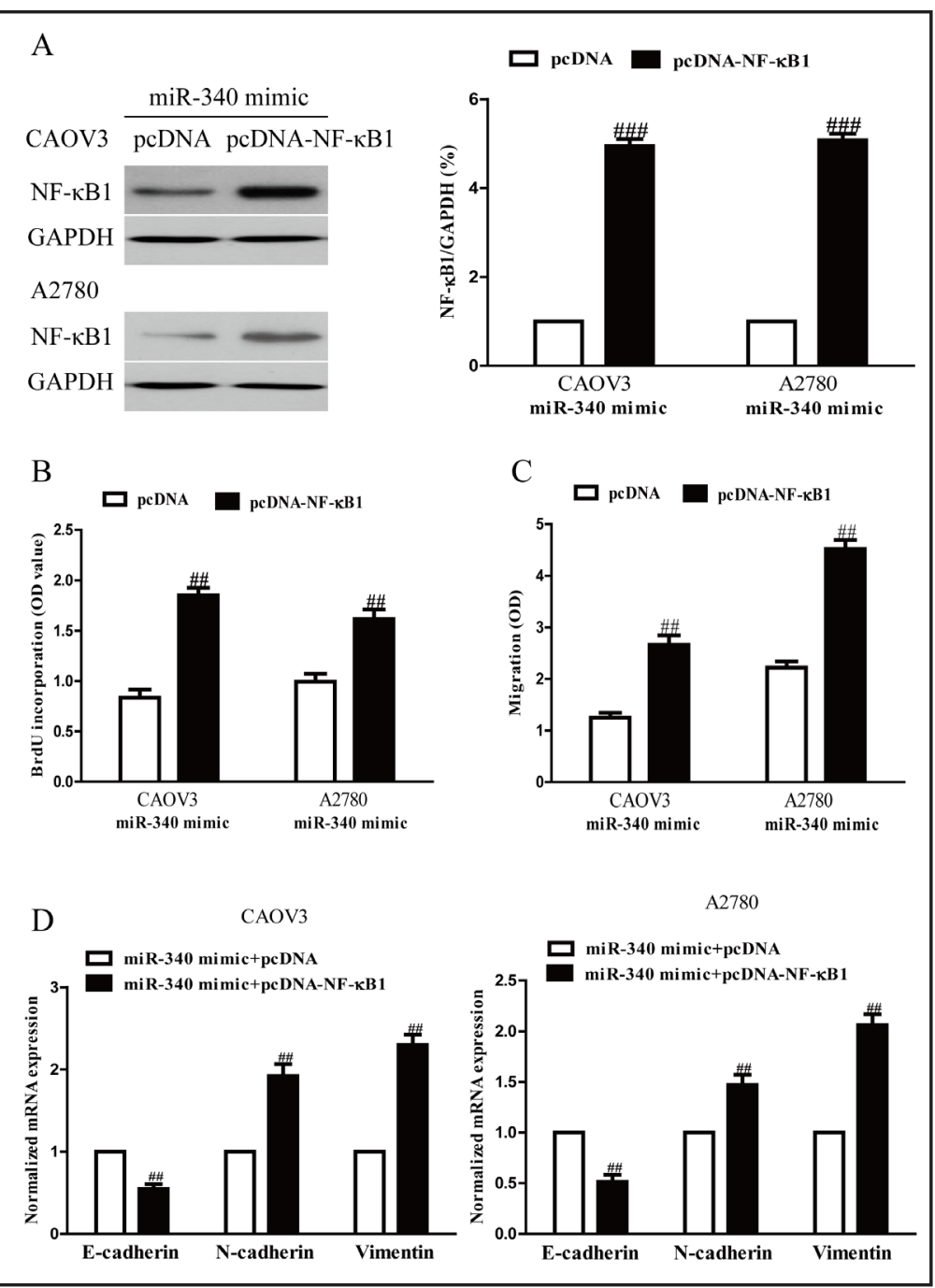

$N F-\kappa B 1$ is a direct target of miR-340 in OC cells

Because NF- $\kappa$ B1 was a binding target of miR-340 predicted by the TargetScan 6.2 , the protein expression of NF- $\kappa \mathrm{B} 1$ was determined by Western blotting in CAOV3 and A2780 cells transfected with miR-340 mimic. Our results demonstrated that NF- $\kappa$ B1 expression was apparently decreased after up-regulation of miR-340 (Fig. 6A). To further confirm whether a direct target of miR-340 was NF- $\kappa$ B1, $3^{\prime}$-UTR of NF- $\kappa$ B1 was cloned into a luciferase reporter vector and the putative miR-340 binding site in the NF- $\kappa$ B1 $3^{\prime}$-UTR was mutated (Fig. 6B). Luciferase reporter assay was used to determine the effect of miR-340 mimic. Our data displayed that introduction of miR-340 dramatically suppressed the luciferase activity of pMir-NF- $\kappa$ B1 3'-UTR WT (Fig. 6C). The effect of miR-340 was abolished by mutation of the miR-340-binding site in the NF- $\kappa$ B1 3'-UTR, which suggested that miR-340 directly and negatively regulated NF- $\kappa \mathrm{B} 1$.

Suppression of NF-KB1 is essential for miR-340 mimic-inhibited cell proliferation and invasion in OC cells

To confirm whether miR-340 inhibited the proliferation, invasion and EMT of OC cells in a NF- $\kappa$ B1-dependent manner, we cotransfected CAOV3 and A2780 cells with miR340 mimic and pcDNA3.1-NF- $\kappa$ B1 vector (Fig. 7A). Data from Brdu-ELISA assay showed that overexpression of NF- $\kappa$ B1 in cells transfected with the miR-340 mimic promoted proliferation of OC cells (Fig. 7B). The Transwell assay showed that up-regulation of NF- 


\section{Cellular Physiology Cell Physiol Biochem 2016;38:1915-1927 \\ \begin{tabular}{c|c|c|} 
DOI: 10.1159/000445553 & $\begin{array}{l}\text { O } 2016 \text { The Author(s). Published by S. Karger AG, Basel } \\
\text { www.karger.com/cpb }\end{array}$ \\
\cline { 2 - 3 } and Biochemished online: May 09, 2016 &
\end{tabular} \\ Li/Sun/Liu: MiR-340 Inhibits Growth and Metastasis of OC Cells by NF-KB1 Inactivation}

$\kappa \mathrm{B} 1$ could reverse the inhibitory effect of the miR-340 mimic on invasion of OC cells (Fig. $7 \mathrm{C})$. Moreover, overexpression of NF- $\kappa \mathrm{B} 1$ decreased E-cadherin expression of the epithelial marker, and increased $\mathrm{N}$-cadherin and Vimentin expressions of the mesenchymal markers at mRNA levels in CAOV3 and A2780 cells transfected with miR-340 mimic (Fig. 7D). Therefore, the inhibitory effects of miR-340 were reversed by up-regulation of NF- $\kappa$ B1. Our finding clearly confirmed that miR-340 suppressed cell proliferation, invasion and EMT in OC cells by decreasing NF- $\kappa \mathrm{B} 1$, and that decreased NF- $\kappa \mathrm{B} 1$ was essential for the miR-340-induced inhibitory effects in $\mathrm{OC}$ cells.

\section{Discussion}

In recent years, many reports have shown the miRNAs are dysregulated in multiple types of cancers [22-24]. It is critical for identification of cancer-specific miRNAs and their targets to understand their role in oncogenesis and define novel therapeutic targets [25-27]. In this paper, we focused on the function of miR-340 in the pathogenesis of human ovarian cancer. Firstly, we detected miR-340 level in OC cell lines by RT-PCR assay. We found that the level of miR-340 was significantly reduced in cancer cells compared to the normal human fallopian tube epithelial cell line FTE187.

Many reports on the function of miR-340 down-regulation in human tumorigenesis have been showed. Interestingly, making use of miRNA expression analysis and RT-PCR, we also found that level of miR-340 was significantly reduced in ovarian cancer cells compared to relevant normal cells [28]. In addition, several reports have demonstrated that miR340 was also evidently down-regulated in colorectal cancer, breast cancer, glioblastoma and oral squamous cell carcinoma [29-32]. Therefore, aberrant regulation of miR-340 was altofrequent of in multiple types of cancer cells and tissues, indicating that decreased miR340 might play a critical role in tumorigenesis.

It has been presumed that many miRNAs were decreased in cancers, suggesting that they may normally function as tumor suppressor genes. Hence, we hypothesized that miR-340 was an inhibitory factor of growth in human ovarian cancer cells. Because level of miR-340 was down-regulated in cancer cells and tissues, we expected that up-regulation of miR-340 would lead to arresting cell growth. Using the Brdu-ELISA assay, we found that CAOV3 and A2780 cells transfected with the miR-340 mimic exhibited decreased growth compared to cells transfected with miR-NC. Cell cycle analyses also showed that the percentage of cells in the G1-phase was increased and the percentage of cells in the S-phase was decreased in cells transfected with miR-340 mimic compared to cells transfected with miR-NC. Moreover, flow cytometry analysis demonstrated that miR-340 mimic could evidently induced apoptosis of CAOV3 and A2780 cells compared with miR-NC group. It has been well known that cell cycle progression and apoptosis are regulated by numerous proteins. In addition, Transwell assay showed that miR-340 mimic dramatically inhibited the invasion of CAOV3 and A2780 cells compared with miR-NC group. Furthermore, we determined the change of EMT markers in CAOV3 and A2780 cells transfected with miR-24 mimic. Our results showed that upregulation of miR-340 could markedly suppress invasive ability of BC cells by dramatically up-regulating the epithelial marker E-cadherin and down-regulating the mesenchymal marker N-cadherin and Vimentin, which supported that miR-340 might suppress EMT process to restrain cell invasion and metastasis.

It has been reported that miR-340 affects cell growth, metastasis and apoptosis of cancer cells possibly by targeting CDK6, ROCK1 and cMet [31, 33, 34]. Although bioinformatic tools may help to reveal putative mRNA targets of miRNAs, experimental procedures are required for their validation. In our paper, we show that miR-340 targeted the NF- $\kappa B 1 \mathrm{mRNA}$, thus revealing a possible mechanism associated with ovarian oncogenesis.

Actually, NF- $\kappa B 1$, a member of the Rel/NF- $\kappa B$ transcription factor family, plays critical roles in the regulation of immune responses, embryo and cell lineage development, cell-cycle progression, cell apoptosis, and tumorigenesis [35-37]. Our data showed that NF- $\kappa$ B1 was a 


\section{Cellular Physiology Cell Physiol Biochem 2016;38:1915-1927 \\ \begin{tabular}{c|c|c|} 
DOI: 10.1159/000445553 & $\begin{array}{l}\text { O } 2016 \text { The Author(s). Published by S. Karger AG, Basel } \\
\text { www.karger.com/cpb }\end{array}$ \\
\hline Published online: May 09, 2016
\end{tabular} \\ Li/Sun/Liu: MiR-340 Inhibits Growth and Metastasis of OC Cells by NF-KB1 Inactivation}

target of miR-340. First, using western blotting, we confirmed that introduction of miR-340 could cause the significant decrease in NF- $\kappa B 1$ protein level. In addition, we found that the ability of miR-340 to regulate NF- $\mathrm{kB1}$ expression was direct, because it bound to the 3'UTR of NF- $\kappa B 1$ mRNA with complementarity to the miR-340 seed region. Moreover, the luciferase activity of NF- $\mathrm{BB} 13$ '-UTR was specifically responsive to miR-340 up-regulation. However, mutation of the miR-340 binding site abolished the effect of miR-340 on the regulation of luciferase activity. In this study, overexpression of NF- $\kappa B 1$ could also rescue OC cells from inhibition of cell growth, invasion and EMT caused by miR-340. However, the underlying mechanisms by which NF- $\kappa B 1$ affects growth, invasion and EMT of OC cells remain to be established.

NF- $\kappa$ B1 facilitates invasion and metastasis of cancer cells partly by transcriptional regulation of MMP-2, MMP-9 and E-cadherin. Liu et al have demonstrated that miR-9 suppressed migration and invasion of uveal melanoma cells in part through direct targeting NF- $\mathrm{kB} 1$ expression and down-regulation of its downstream molecules MMP-2 and -9 [38]. Another report has shown that miR-9 could inhibit metastasis of melanoma cells by suppression of E-cadherin expression through targeting NF- $\kappa B 1$ [39]. In our paper, we found that MMP-2 and MMP-9 altered in the same pattern as NF- $\kappa B 1$, but E-cadherin altered in the reverse pattern as NF- $\mathrm{B} 1$. Therefore, NF- $\mathrm{KB} 1$-regulated MMP-2, MMP-9 and E-cadherin may contribute to miR-340-suppressed invasion and metastasis of OC cells.

In summary, we showed that miR-340 was evidently decreased in OC cells. Introduction of miR-340 inhibited proliferation, invasion, EMT and induced apoptosis of OC cells through directly targeting NF- $\kappa \mathrm{B} 1$. This novel miR-340/NF- $\kappa$ B1 axis might provide new insights into the molecular mechanisms underlying progression and metastasis of $\mathrm{OC}$, and overexpression of miR-340 might be a potential therapeutic strategy for the treatment of OC in the future.

\section{Disclosure Statement}

The authors have no competing financial interests to disclose.

\section{References}

1 Corney DC, Nikitin AY: MicroRNA and ovarian cancer. Histol Histopathol 2008;23:1161-1169.

2 Bartel DP: Micrornas: genomics, biogenesis, mechanism, and function. Cell 2004;116:281-297.

3 Lee RC, Ambros V: An extensive class of small RNAs in Caenorhabditis elegans. Science 2001;294:862-864.

4 Kim VN: MicroRNA biogenesis: coordinated cropping and dicing. Nat Rev Mol Cell Biol 2005;6:376-385.

5 Li YC, Li CF, Chen LB, Li DD, Yang L, Jin JP, Zhang B: MicroRNA-766 targeting regulation of SOX6 expression promoted cell proliferation of human colorectal cancer. Onco Targets Ther 2015;8:2981-2988.

6 Hua K, Yang W, Song H, Song J, Wei C, Li D, Fang L: Up-regulation of miR-506 inhibits cell growth and disrupt the cell cycle by targeting YAP in breast cancer cells. Int J Clin Exp Med 2015;8:12018-12027.

7 Li Y, Zu L, Wang Y, Wang M, Chen P, Zhou Q: miR-132 inhibits lung cancer cell migration and invasion by targeting SOX4. J Thorac Dis 2015;7:1563-1569.

8 Teng Y, Zhao L, Zhang Y, Chen W, Li X: Id-1, a protein repressed by miR-29b, facilitates the TGFß1-induced epithelial-mesenchymal transition in human ovarian cancer cells. Cell Physiol Biochem 2014;33:717-730.

9 Ge T, Yin M, Yang M, Liu T, Lou G: MicroRNA-302b suppresses human epithelial ovarian cancer cell growth by targeting RUNX1. Cell Physiol Biochem 2014;34:2209-2220.

10 Chen Q, Qin R, Fang Y, Li H: Berberine Sensitizes Human Ovarian Cancer Cells to Cisplatin Through miR-93/ PTEN/Akt Signaling Pathway. Cell Physiol Biochem 2015;36:956-965.

11 Song N, Liu H, Ma X, Zhang S: Placental growth factor promotes metastases of ovarian cancer through MiR543-regulated MMP7. Cell Physiol Biochem 2015;37:1104-1112.

12 Fang Y, Xu C, Fu Y: MicroRNA-17-5p induces drug resistance and invasion of ovarian carcinoma cells by targeting PTEN signaling. J Biol Res (Thessalon) 2015;22:12. 


\section{Cellular Physiology Cell Physiol Biochem 2016;38:1915-1927 and Biochemistry \begin{tabular}{c|c} 
DOI: 10.1159/000445553 & $\begin{array}{l}\text { O 2016 The Author(s). Published by S. Karger AG, Basel } \\
\text { www.karger.com/cpb }\end{array}$ \\
\cline { 2 - 3 }
\end{tabular}}

Li/Sun/Liu: MiR-340 Inhibits Growth and Metastasis of OC Cells by NF-kB1 Inactivation

13 Niu K, Shen W, Zhang Y, Zhao Y, Lu Y: MiR-205 promotes motility of ovarian cancer cells via targeting ZEB1. Gene 2015;574:330-336.

14 Zhu T, Yuan J, Wang Y, Gong C, Xie Y, Li H: MiR-661 contributed to cell proliferation of human ovarian cancer cells by repressing INPP5J expression. Biomed Pharmacother 2015;75:123-128.

15 Ye Z, Zhao L, Li J, Chen W, Li X: miR-30d Blocked Transforming Growth Factor $\beta 1$-Induced EpithelialMesenchymal Transition by Targeting Snail in Ovarian Cancer Cells. Int J Gynecol Cancer 2015;25:15741581.

16 Liu X, Ma L, Rao Q, Mao Y, Xin Y, Xu H, Li C, Wang X: MiR-1271 Inhibits Ovarian Cancer Growth by Targeting Cyclin G1. Med Sci Monit 2015;21:3152-3158.

17 Guo T, Yu W, Lv S, Zhang C, Tian Y: MiR-302a inhibits the tumorigenicity of ovarian cancer cells by suppression of SDC1. Int J Clin Exp Pathol 2015;8:4869-4880.

18 Zhao H, Li M, Li L, Yang X, Lan G, Zhang Y: MiR-340 is down-regulated in human osteosarcoma and inhibits osteosarcoma cells proliferation, migration and invasion, and promotes apoptosis. PLoS One 2013;8:e83571.

19 Wei P, Qiao B, Li Q, Han X, Zhang H, Huo Q, Sun J: microRNA-340 Suppresses Tumorigenic Potential of Prostate Cancer Cells by Targeting High-Mobility Group Nucleosome-Binding Domain 5. DNA Cell Biol 2016;35:33-43.

20 Yan S, Jiang H, Fang S, Yin F, Wang Z, Jia Y, Sun X, Wu S, Jiang T, Mao A: MicroRNA-340 Inhibits Esophageal Cancer Cell Growth and Invasion by Targeting Phosphoserine Aminotransferase 1. Cell Physiol Biochem 2015;37:375-386.

21 Fernandez S, Risolino M, Mandia N, Talotta F, Soini Y Incoronato M, Condorelli G, Banfi S, Verde P: miR-340 inhibits tumor cell proliferation and induces apoptosis by targeting multiple negative regulators of p27 in non-small cell lung cancer. Oncogene 2015;34:3240-3250.

22 Calin GA, Dumitru CD, Shimizu M, Bichi R, Zupo S, Noch E, Aldler H, Rattan S, Keating M, Rai K, Rassenti L, Kipps T, Negrini M, Bullrich F, Croce CM: Frequent deletions and down-regulation of micro-RNA genes miR15 and miR16 at 13q14 in chronic lymphocytic leukemia. Proc Natl Acad Sci U S A 2002;99:1552415529.

23 Metzler M, Wilda M, Busch K, Viehmann S, Borkhardt A: High expression of precursor microRNA-155/BIC RNA in children with Burkitt lymphoma. Genes Chromosomes Cancer 2004;39:167-169.

24 Eis PS, Tam W, Sun L, Chadburn A, Li Z, Gomez MF, Lund E, Dahlberg JE: Accumulation of miR-155 and BIC RNA in human B cell lymphomas. Proc Natl Acad Sci U S A 2005;102:3627-3632.

25 Calin GA, Liu CG, Sevignani C, Ferracin M, Felli N, Dumitru CD, Shimizu M, Cimmino A, Zupo S, Dono M, Dell'Aquila ML, Alder H, Rassenti L, Kipps TJ,Bullrich F, Negrini M, Croce CM: MicroRNA profiling reveals distinct signatures in B cell chronic lymphocytic leukemias. Proc Natl Acad Sci U S A 2004;101:1175511760.

26 Lu J, Getz G, Miska EA, Alvarez-Saavedra E, Lamb J, Peck D, Sweet-Cordero A, Ebert BL, Mak RH, Ferrando AA, Downing JR, Jacks T, Horvitz HR, Golub TR: MicroRNA expression profiles classify human cancers. Nature 2005;435:834-838.

27 Volinia S, Calin GA, Liu CG, Ambs S, Cimmino A, Petrocca F, Visone R, Iorio M, Roldo C, Ferracin M, Prueitt RL, Yanaihara N, Lanza G, Scarpa A, Vecchione A,Negrini M, Harris CC, Croce CM: A microRNA expression signature of human solid tumors defines cancer gene targets. Proc Natl Acad Sci U S A 2006;103:22572261.

28 De Cecco L, Berardi M, Sommariva M, Cataldo A, Canevari S, Mezzanzanica D, Iorio MV, Tagliabue E, Balsari A: Increased sensitivity to chemotherapy induced by CpG-ODN treatment is mediated by microRNA modulation. PLoS One 2013;8:e58849.

29 Takeyama H, Yamamoto H, Yamashita S, Wu X, Takahashi H, Nishimura J, Haraguchi N, Miyake Y, Suzuki R, Murata K, Ohue M, Kato T, Takemasa I,Mizushima T, Ishii H, Mimori K, Doki Y, Mori M: Decreased miR-340 expression in bone marrow is associated with liver metastasis of colorectal cancer. Mol Cancer Ther 2014; 13: 976-985.

30 Chen CP, Sun ZL, Lu X, Wu WX, Guo WL, Lu JJ, Han C, Huang JQ, Fang Y: miR-340 suppresses cell migration and invasion by targeting MYO10 in breast cancer. Oncol Rep 2015;35:709-716.

31 Li X, Gong X, Chen J, Zhang J, Sun J, Guo M: miR-340 inhibits glioblastoma cell proliferation by suppressing CDK6, cyclin-D1 and cyclin-D2. Biochem Biophys Res Commun 2015;460:670-677. 
32 Xu P, Li Y, Zhang H, Li M, Zhu H: MicroRNA-340 Mediates Metabolic Shift in Oral Squamous Cell Carcinoma by Targeting Glucose Transporter-1. J Oral Maxillofac Surg 2016;74:844-850.

33 Zhou X, Wei M, Wang W: MicroRNA-340 suppresses osteosarcoma tumor growth and metastasis by directly targeting ROCK1. Biochem Biophys Res Commun 2013;437:653-658.

34 Wu ZS, Wu Q Wang CQ, Wang XN, Huang J, Zhao JJ, Mao SS, Zhang GH, Xu XC, Zhang N: miR-340 inhibition of breast cancer cell migration and invasion through targeting of oncoprotein c-Met. Cancer 2011;117:28422852.

35 Zandi E, Karin M: Bridging the gap: composition, regulation, and physiological function of the IkappaB kinase complex. Mol Cell Biol 1999;19:4547-4551.

36 Karin M, Ben-Neriah Y: Phosphorylation meets ubiquitination: the control of NF-[kappa]B activity. Annu Rev Immunol 2000;18:621-663.

37 Israël A: The IKK complex: an integrator of all signals that activate NF-kappaB?. Trends Cell Biol 2000;10:129-133.

38 Liu N, Sun Q, Chen J, Li J, Zeng Y, Zhai S, Li P, Wang B, Wang X: MicroRNA-9 suppresses uveal melanoma cell migration and invasion through the NF-кB1 pathway. Oncol Rep 2012;28:961-968.

39 Liu S, Kumar SM, Lu H, Liu A, Yang R, Pushparajan A, Guo W, Xu X: MicroRNA-9 up-regulates E-cadherin through inhibition of NF- KB1-Snail1 pathway in melanoma. J Pathol 2012;226:61-72. 